\title{
CONGENITAL MALFORMATIONS OF THE HEART AND THE GREAT VESSELS ASSOCIATED WITH CONGENITAL ABSENCE OF THE SPLEEN
}

\author{
BY \\ DAVID LEVY \\ From the Department of Paediatrics B, Rambam Government Hospital, Haifa, Israel
}

(RECEIVED FOR PUBLICATION OCTOBER 14, 1956)

The unusual combination of congenital malformations of the heart and great vessels on the one hand, with agenesis of the spleen on the other hand has been looked upon as a great medical curiosity. In the past it had been the pathologist exclusively who from time to time encountered this association of anomalies. More recently, however, cases have been diagnosed by the clinician during the life of these patients.

The first case on record of this combination of anomalies is that of Martin (1826), who described the necropsy findings of a case of septal defects, transposition of the great vessels and agenesis of the spleen. Afterwards a number of other reports dealt with cardiac anomalies curiously associated with agenesis of the spleen. In 1934 Putschar reviewed the findings of all cases reported up to that time, collecting from the literature a total of 22 cases. The next review of the literature is that of Polhemus and Schafer (1952). These authors pointed out for the first time that these cases of cardiac anomalies associated with absence of the spleen actually constitute a definite syndrome. The latest review is that of Biorn Ivemark (1955) who collected all the cases reported in the literature since the very first case of Martin (1826) and added 14 more cases of his own.

The first two cases of agenesis of the spleen associated with cardiac anomalies diagnosed in vivo and confirmed at necropsy were those reported by Gasser and Willi (1952). They based their antemortem diagnosis on a persistently increased number of Heinz bodies in the erythrocytes of the peripheral blood.

Recently a case of agenesis of the spleen with multiple anomalies of the heart and the great vessels was observed in the Paediatric Department B of the Rambam Government Hospital in Haifa. In this paper we wish to report this case. We should also like to discuss the correlation of the clinical signs observed.

\section{Case Report}

S.Y., a white 10-day-old boy, was born in a private maternity clinic after a prolonged labour and with the aid of mid-forceps. On the day after birth the house physician observed that the child was cyanotic and cold. On the fifth day of life a systolic murmur was heard over the apex. The baby was then transferred to this Department for clinical appraisal.

The family history was negative for heart disease or congenital anomalies. The mother was a 22-year-old primipara primigravida. She denied any rashes or serious illnesses during the first trimester of this pregnancy.

When first seen in our Department the baby was found to be well developed and well nourished. His weight was $7.92 \mathrm{lb}$. The lips, ear lobes and nail beds were distinctly cyanotic and the skin had a rather bluish hue. The apex beat of the heart was felt on the right side of the sternum in the fourth intercostal space. The heart sounds were clear and heard best on the right side of the chest. There were no murmurs. The spleen and the liver were not palpable under the costal margin.

Laboratory examinations gave the following results: Hb 15.8 g. \%, red blood cells $3,900,000 / \mathrm{mm}^{3}$, white blood cells $7,800 / \mathrm{mm}^{3}$ (65\% neutrophils, $6 \%$ eosinophils, $1 \%$ basophils, $8 \%$ monocytes, $19 \%$ lymphocytes and $2 \%$ plasma cells). Stools and urine were normal.

A radiograph of the abdomen showed the liver to be normally placed on the right and the stomach on the left. The radiological picture of the heart was considered to indicate dextrocardia confirming the clinical impression. The left lung was shadowed. A control examination a week later revealed shadows in both upper lobes.

An electrocardiogram on the eighth day of his hospital stay showed sinus tachycardia, with a rate of 140 per minute. There was no axis deviation. The heart was in the intermediary position ( $\mathrm{P}-\mathrm{Q}=\mathbf{0} \cdot 12 \mathrm{sec}$.; $\mathrm{QRS}=$ 0.07 sec.; $Q T=0.24$ sec.; $R-P=0.44$ sec.). The $Q$ wave was deep in leads I and II and less in aVF and aVL; the $T$ wave was negative in leads $I$, aVL and biphasic $(-+)$ in lead II. The S-T segment was elevated in V1 and V2, but less than $2 \mathrm{~mm}$.; V1 and V2 showed an RS pattern and V5 qR pattern; the transitional zone 
was between V3 and V4. This was interpreted as indicating a normal position of the heart chambers. The electrocardiographic diagnosis was probable tricuspid atresia, trilocular heart.

During the first week in hospital the patient exhibited intermittent attacks of cyanosis. Repeated physical examinations were considered essentially normal, except for the cyanosis. At no time were any abnormal neurological signs found and no marked respiratory embarrassment was noted.

Throughout this period the general condition of the child was satisfactory and he did not lose weight. His appetite was good. No drugs were administered during this period.

At the beginning of the second week in hospital signs of pneumonia of the left upper lobe were elicited on physical examination. Later on the baby developed diarrhoea and vomiting with secondary disturbances of the fluid and electrolyte balance. He was treated with oxygen, plasma, intravenous fluids and electrolytes, penicillin and streptomycin and oxytetracycline but he continued to do poorly. On the eve of his death a number of small fluctuant nodules appeared on the anterior chest wall. One of them, situated over the upper part of the sternum, showed clear pulsations, synchronous with the heart beat. This nodule was punctured immediately after death and pus withdrawn, and culture showed a pure growth of Proteus vulgaris. On the morning of his 31 st day in hospital the patient expired.

\section{Necropsy Report}

The essential findings at necropsy were as follows: In the peritoneal cavity the abdominal organs were in their normal positions. The liver was asymmetric, the left lobe being bigger than the right lobe. The spleen was entirely absent. There was a common mesentery. In the chest a retrosternal abscess communicating with a subcutaneous abscess through a necrotic zone in the upper part of the sternum was found. The lungs were well aerated and not congested. The left lung was a mirror image of the right lung, there being a middle lobe. The trachea and bronchi were normal in appearance. The heart lay in the right hemithorax, but the apex pointed to the left. There was a left aortic arch with normal branching. The ductus arteriosus was obliterated. There was a purulent pericarditis. There were big septal defects between the two auricles and between the two ventricles. The right atrium was rudimentary. The right ventricle was not developed. The tricuspid valve was atretic. The pulmonary veins formed a common trunk that communicated with the superior vena cava and entered the right atrium. The pulmonary artery arose from the right ventricle. The superior vena cava communicated with the pulmonary vein in the right lung and with the innominate vein which entered the left atrium. The inferior vena cava entered the right atrium. The aorta arose from the left ventricle. The abdominal aorta descended normally on the left and the inferior vena cava on the right (Fig. 1). There was passive congestion of the abdominal organs.

\section{Discussion}

The post-mortem diagnoses of this case can be classified into two groups. On the one hand there were multiple congenital anomalies consisting of various malformations of the heart and agenesis of the spleen. On the other hand there were various manifestations of septicaemia. Whereas the signs and symptoms of septicaemia were readily recognized during life, the symptoms and signs of the heart anomalies were partly misinterpreted and diagnosed only at necropsy.

The occurrence of septicaemia in this case as well as the lack of response to adequate quantities of broad-spectrum antibiotics deserve special attention. A review of the pertinent literature seems to indicate that in many of the reported cases very severe purulent infections have occurred. Thus Ivemark (1955) found in eight of his 14 patients signs of purulent infections at post-mortem examinations.

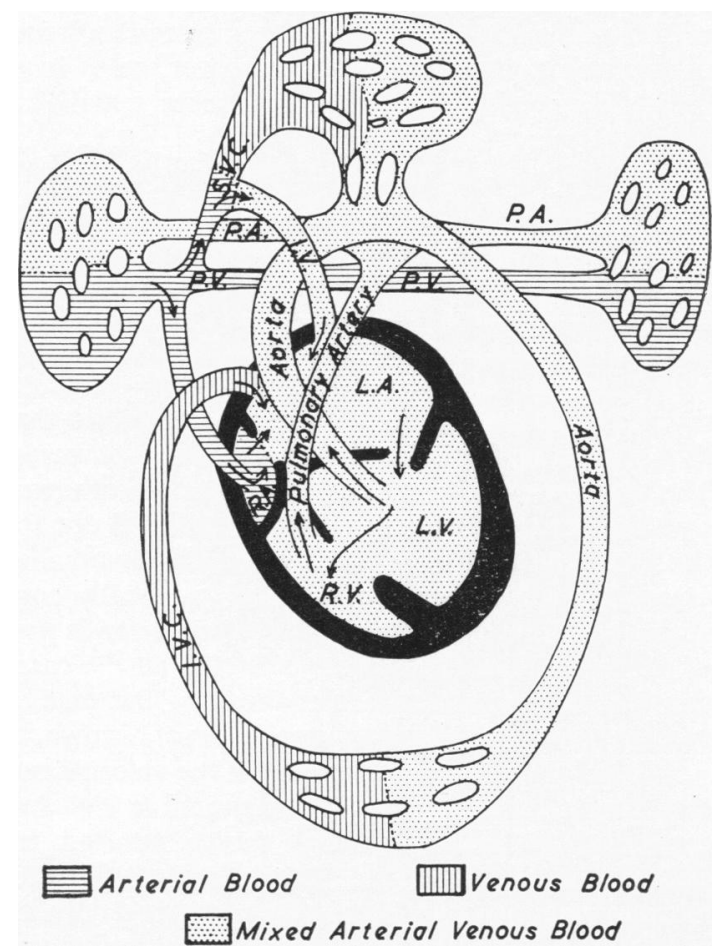

FIG. 1.-Diagrammatic picture of the heart and great vessels in a case with associated absence of the spleen.

$$
\begin{aligned}
& \text { R.V. = right ventricle } \\
& \text { R.A. = right auricle } \\
& \text { L.V. = left ventricle } \\
& \text { L.A. = left auricle } \\
& \text { I.V. = innominate vein } \\
& \text { P.A. = pulmonary artery } \\
& \text { P.V. = pulmonary vein } \\
& \text { S.V.C. = superior vena cava } \\
& \text { I.V.C. = inferior vena cava }
\end{aligned}
$$


Other cases of congenital absence of the spleen showing evidence of severe purulent infection at the time of death have been reported by Polhemus and Schafer (1952), Gardère (1908), McLean and Craig (1922), Feldmann (1930) and Leikin (1951). Ramsay (1850) reported a case of persistent gastro-enteritis of 12 months' duration in which at necropsy no spleen was found.

It seems, moreover, that not only does congenital absence of the spleen apparently predispose these young infants to infection, but surgical removal of the spleen in young infants is also accompanied by the same risk. Thus King and Shumacker (1952) pointed out that after splenectomy in infants under the age of 6 months septicaemia very frequently develops. Gellis (1954) reported three cases in which septicaemia developed several months after splenectomy. Hoefnagel (1956) reported two cases of repeated severe infections which ultimately led to their death in two brothers whose spleens were removed for idiopathic thrombocytopenic purpura.

An explanation of this relationship of purulent infection to the absence of the spleen is at present still conjectural. However, there is ample experimental evidence available to suggest that the spleen has a definite role in the defence mechanism of the body, probably through antibody formation. Thus in rodents removal of the spleen interferes with the development of an immune response (Rowley, 1950a). The same author has also demonstrated, in human beings who have undergone splenectomy, a failure to respond with a significant rise in antibody titre after the administration of an antigen (Rowley, 1950b). Jacobson and Robson (1952) have shown that shielding with lead the spleen in rabbits during total body irradiation prevents the usual reduction in these circumstances of circulating antibody concentration.

On the basis of the above evidence it seems fair to conclude that, unlike conditions in adults, the spleen of young infants plays a major role in the defence of the body against infection while other defence organisms at this age are apparently underdeveloped. This would explain the fact emphasized by King and Shumacker (1952) that patients whose spleen has been removed before the age of 6 months are relatively more prone to infection than older children undergoing splenectomy.

Serious infections in a cyanotic infant, even without clear-cut evidence of heart disease, should, therefore, serve as indications for the search for co-existent asplenia. When such a combination of cyanosis and persistent infections is observed, further studies should be performed. The chief aid in the diagnosis of this condition is derived from haematological examinations. A persistently increased number of Heinz bodies in the erythrocytes of the peripheral blood has been pointed out by Gasser and Willi (1952) as a constant feature in this syndromc. Bush and Ainger (1955) performed detailed studies in a case later proved to be one of agenesis of the spleen. They found that after the intravenous administration of twice the amount of iron necessary to increase haemoglobin to $18 \mathrm{~g}$. target cells persisted, Howell-Jolly bodies were present throughout the clinical course, normoblasts that were present before the iron treatment increased sixfold after it, siderocytes appeared immediately after therapy and persisted and the leucocyte count was elevated throughout the clinical observation without cause. The osmotic fragility of the red blood cells was decreased.

It must be borne in mind, however, that the appearance of Heinz bodies or Howell-Jolly bodies does not in itself constitute conclusive evidence of asplenia. Gasser (1954) reported a Heinz body anaemia after topical application of 'resorcinol' in a case of infantile eczema. Heinz bodies are also found after the administration of methylene blue as well as in certain haemolytic anaemias. A full haematological examination, similar to the one performed by Bush and Ainger (1955) is, therefore, indicated in cases of suspected asplenia in order to reach an ante-mortem diagnosis.

\section{Summary}

A case of congenital absence of the spleen with associated multiple anomalies of the heart and the great vessels presented various manifestations of severe septicaemia unresponsive to adequate quantities of antibiotics. A review of the pertinent literature seems to indicate that a causal relationship exists between the agenesis of the spleen and the very severe infections observed in these cases. It is suggested that serious infections in a cyanotic infant, even without clear-cut evidence of heart disease, should lead to a search for co-existent asplenia.

My thanks are due to Dr. W. Falk, Head of the Department of Paediatrics B, Rambam Government Hospital, and to Dr. A. Friedman for their help and encouragement in preparing this paper; the pathological examination was performed by Dr. B. Gellei, Head of the Pathological Department of the Rambam Government Hospital; the electrocardiographic examinations were performed by Drs. Y. Heichman and A. Askireli.

\section{REFERENCES}

Bush, J. A. and Ainger, L. E. (1955). Pediatrics, 15, 93.

Feldmann. B. A. (1930). Jb. Kinderkeilk., 129, 221.

Garčèe (1908). Lyon méd., 110, 897. Cited by Ivemark B. I. (1955). 
Gasser, C. (1954). Helv. paediat. Acta, 9, 285.

and Willi, H. (1952). Ibid., 7, 369 .

Gellis, S. S. (1954), Year Book of Pediatrics, 1954-55, Editor's comment 275.

Hoefnagel, R. (1956). Clin. Proc. Child. Hosp. (Wash.), 12, 48.

Ivemark, B. I. (1955). Acta paediatr. (Uppsala), 44, suppl. 104.

Jacobson, L. O. and Robson, M. J. (1952). J. Lab. clin. Med., 39, 169.

King, H. and Shumacker, H. B., Jr. (1952). Ann. Surg., 136,

Leikin, S. L. (1951). Clin. Proc. Child. Hosp. (Wash.), 7, 236.
Martin, G. (1826). Bull. Soc. anat. Paris, 1, 39. Cited by Ivemark, B. I. (1955)

McLean, S. and Craig, H. R. (1922). Amer. J. med. Sci., 164, 703. Cited by Ivemark, B. I. (1955)

Polhemus, D. W. and Schafer, W. B. (1952). Pediatrics, 9, 696.

Putschar, W. (1934). In Die Morphologie der Missbildungen des Menschen und der Tiere, ed. Schwalbe, E., teil 3, abt. 3, pp. 759-856. Fischer, Jena. Cited by Ivemark, B. I. (1955).

Ramsay, H. A. (1850). Charleston med. J. Rev., 5, 728.

Rowley, D. A. (1950a). J. Immunol., 64, 289.

(1950b). Ibid., 65, 515 . 